\title{
Simulation of Non-Axisymmetric Floating Zone Crystal Growth under Microgravity
}

The possibility of growing crystals of non-circular cross-section by using the floating zone technique is considered. basing the analysis on the isothermal liquid bridge model, and restricting the study to a linear asymptotic analysis.

An almost square initial seed is considered in more detail, although the analysis is general and can be applied to other initial shapes, not only of the seed but also of the supply rod, that can be of any cross-section.

The influence of non-circular seed and/or feed cross-section shape, and of a possible axial acceleration typical of microgravity platforms (sounding rockets and space labs), on both the liquid interface shape and the solid grown shape, are analysed, including the stability limits by breaking of the molten bridge.

\section{Introduction}

As it is well known, in the floating zone technique of crystal growth, a molten zone is established between two rods: a charge or feed rod of polycrystalline material and a monocrystalline seed rod. The molten zone can be established in a variety of ways depending on the material to be crystallised; for example radio frequency induction heating using a single turn coil or radiation heating in mirror furnaces are commonly used with silicon whereas electron beam melting is employed in the case of refractory metals.

On earth, gravitational forces set an upper limit to the stable molten zone length and therefore to the crystal diameter, so that rods of only a few millimetres in diameter could be easily zoned, although some techniques have been devised [1], based on a precise knowledge of the behaviour of the liquid phase, that allow the growing of cylindrical shaped crystals with diameters of several centimetres. These constraints partly disappear in a microgravity environment, like the one existing in orbital laboratories, where the crystal growth can be easier and better studied. In fact several experiments dealing with crystal growth by using the floating zone technique have been performed on board orbital platforms [2].

The equilibrium shapes and stability limits of the floating zone melt under the large variety of disturbances that could arise either accidentally or intentionally during the growing process is a matter of great concern. Its study involves a formidable task both because of the material characteristics of the melt, whose properties are strongly temperature dependent, and because of the complexities associated to the disturbances which could be imposed on the zone. Thence, several simplifications must be introduced in the model. The simplest approach consists in disregarding phase changes, considering an isothermal liquid zone held between two parallel solid disks, the socalled liquid bridge model [3].

of course there are many differences between isothermal liquid bridges and real floating zones. The behaviour of the melt in a real floating zone is driven by many effects (dynamics, convection, diffusion, phase changes, electric and magnetic effects, reactive processes,...) which are not accounted for in the liquid bridge problem. However, in spite that the real solid-liquid interfaces are rather different between liquid bridges (where supports are assumed to be flat) and floating zones (where protruding solid ends appear), it is of no importance at all for the mechanical stability of the melt. Thus, to a first approximation, assuming that the interest is focussed on the hydrostatic behaviour of the molten zone (equilibrium shapes and their stability), the above-mentioned effects can be considered negligible, valid results are obtained [4], and consequently its use has widespread [5].

Hence, a liquid bridge can be considered as the simplest idealisation of the real configuration appearing in the floating zone technique used for crystal growth and purification of high melting point materials. Equilibrium shapes and stability limits of capillary liquid bridges have been extensively analysed from 
both the theoretical and the experimental point of view during the last decades, and as already said many papers dealing with such fluid configurations have been published (a review of the state of the art in the field can be found in [6-7]).

Although some works considering non-circular supports have been published on crystal growing by the Czochralski method [8-10] and on capillary jets emerging from non-circular nozzles, most of the literature deals with liquid bridges and floating zones between circular disks, with the liquid interface shape being non-axisymmetric or axisymmetric depending on lateral forces or eccentricity being considered or not.

A method to compute the temporal evolution of the molten zone and the melting and resolidifying solid boundary is devised in this paper. The algorithm allows not only to predict the shape of the melted zone at any time and of the grown crystal but to monitor the stability of the process. This way it is possible to select appropriate parameters to ensure a stable growing process.

The steps followed by the algorithm to get the temporal evolution of molten shapes during crystal growth are: first, an initial configuration is assumed, e.g. a liquid bridge spanning between a square solid on one side and a circular solid on the other end, or a solid bar molten in some length. Then, the shape of the free-floating liquid interface in this initial configuration is computed. Afterwards, the growth starts, being modelled by a constant growth angle for the solid slope relative to the liquid slope [11]. The process is iterated until the desired growth length is reached or a necking instability appears.

\section{Modelling}

In the analysis, the grown crystal and the floating zone are represented respectively as a solid mass and a liquid mass whose shapes are given in cylindrical coordinates by $R_{S}(Z, \theta)$ and $R_{F}(Z, \theta)$, respectively, where $R$ stands for the radius, $Z$ and $\theta$ for the axial and azimuthal coordinates, respectively, and the subscripts $S$ and $F$ mean solid and fluid, respectively. To analyse the fluidmechanical aspects of the molten zone, the liquid can be considered limited by two planar solid faces to the borders of which it attaches without slipping, as demonstrated experimentally [12]. A sketch is shown in Fig. 1 for the particular case of a square solid that is melted and then grown.

Two coordinate reference frames will be used: a fix one (say fixed to the grown solid) where the floating zone process would be assumed to take place, and a moving one centred at mid height of the molten free surface. The final data will be presented in the fix reference, whereas the analysis and computation of the free surface shape of the liquid is performed in the moving axis.

Although the linear analysis of the interface shapes of liquid bridges between circular supports and nearly-cylindrical shapes is well documented elsewhere [13], the peculiarities of the noncylindrical supports case here require a detailed explanation because of the new boundary conditions. The keypoint in this

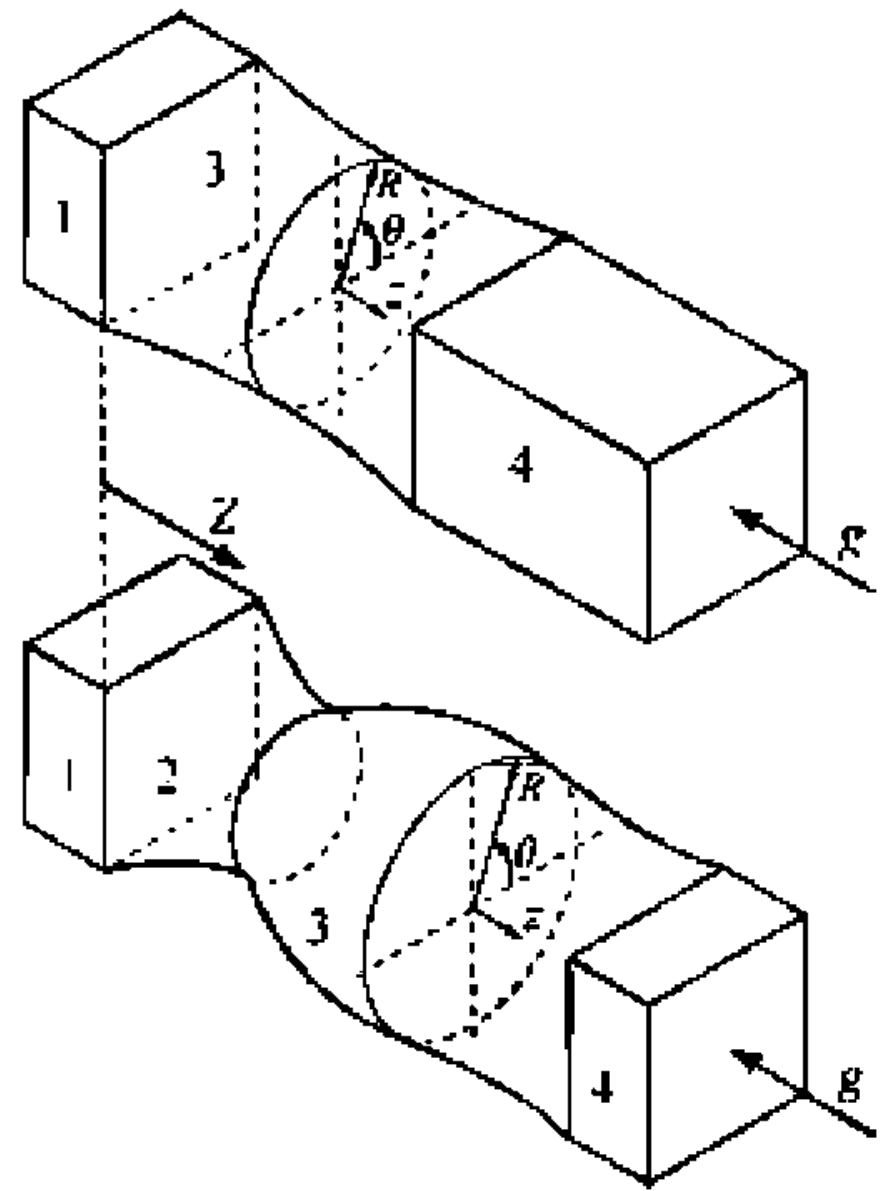

Fig. 1. Geometry of a possible geometric configuration: 1 , seed; 2 , grown crystal: 3 , melt: 4 , feed. A fix reference frame is used to describe the grown solid. and a moving reference frame, centred at the molten length and locally re-scaled is used to describe the liquid interface.

linear analysis is to make the approximation:

$$
R_{F}(Z, \theta)=R_{j}+R_{f}(Z, \theta) \text { with } R_{f} \ll R_{j} .
$$

\section{1 Approximation of the shapes of the solid ends}

The shape (planar cross-section) of the two solid ends may be described in polar coordinates as $R_{i}=R_{i}(\theta)$ and expanded in Fourier cosine-series around a mean radius in the general form (only molten shapes with a symmetry plane are considered and thus, there is no need to include terms in $\sin (j \theta)$ ):

$$
R_{i}(\theta)=R_{i j}+\sum_{j=1}^{\infty} C_{i j} \cos (j \theta),
$$


where the subscript $i=1$ refers to the left-end (seed) and the subscript $i=2$ to the right-end (feed), see Fig. 1, and, from the Fourier expansion:

$$
R_{i j}=\frac{1}{2 \pi} \int_{j}^{2 \pi} R_{i}(\theta) d \theta \quad \text { and } \quad C_{i j}=\frac{1}{\pi} \int_{0}^{2 \pi} R_{i}(\theta) \cos (j \theta) d \theta
$$

This general approximation, valid for any cross-section shape, will be later reduced (for further simplification in the analysis) in the application here presented of near-square shapes to only two terms in the expansion series (2) to measure the non-circularity: a mean radius $\left(R_{10}\right.$ and $R_{20}$ ) and only one coefficient (a single harmonic, $\mathrm{C}_{1 j}$ and $\mathrm{C}_{2 j}$ ), with $j$ being an integer that shows how many lobes bend out of the circle. The justification of using such a simple approximation (only one cosine term) is that more additional terms tend to increase the singular character of the curvature at the vertex of a perfect polygonal shape and would require a cumbersome non-linear analysis with little further teaching.

As said before, and just to be more explicit, the case of a square cross-section is further analysed, i.e. $j=4$, valid for nearsquare cross-section at both ends (untwisted, because no phase difference is retained, although it is a simple matter to include such a possibility). Moreover, the first harmonic for a perfect square shape

$$
R(\theta)=\frac{1}{|\cos (\theta)|+|\sin (\theta)|}
$$

is $C_{i 4} / R_{i 0}=0.14$, according to (3), and this fact further substantiate the simplification to be done.

\section{2 Approximation of the shapes of the liquid interface}

We look for the equilibrium shape of a given volume of liquid $\left(V_{F}\right)$ of constant density $(\rho)$ and surface tension $(\sigma)$, spanning and anchored to arbitrary solid ends a distance $L$ apart, characterised by their mean radius and ripple, $R_{10}$ and $C_{1 j}$ for one end, and $R_{20}$ and $C_{2 j}$ for the other end, and subjected to the sole applied force of a small constant axial acceleration $g$ (microgravity), as indicated in Fig. 1.

To write down the problem formulation in dimensionless form, the characteristic length $R_{0}=\left(R_{20}+R_{10}\right) / 2$ is introduced, and the non-dimensional variables defined in Table 1.

Equilibrium shapes of liquid bridges are described by the Young-Laplace equation, which in dimensionless variables reads (leaving apart electric and magnetic effects):

$$
M(F)+P-B z=0,
$$

where $M(F)$ is twice the mean curvature of the interface, $F(z, \theta)$, that is:

$$
\begin{aligned}
& M(F)= \\
& \frac{F\left[1+\left(F_{z}\right)^{2}\right]\left[F_{\theta \theta}-F\right]+F F_{z z}\left[F^{2}+\left(F_{\theta}\right)^{2}\right]-2 F_{\theta}\left[F_{\theta}+F F_{z} F_{z \theta}\right]}{\left\{F^{2}\left[1+\left(F_{z}\right)^{2}\right]+\left(F_{\theta}\right)^{2}\right\}^{\frac{3}{2}}}
\end{aligned}
$$

and $P$ is a constant related to the difference between the outer pressure and the inner pressure at $z=0$, which has been made dimensionless with $\sigma R_{0}, B$ is the Bond number, defined in Table 1, and subscripts $z$ and $\theta$ indicate derivatives with respect to $z$ and $\theta$, respectively.

The dimensionless boundary conditions at the disks are:

$$
\begin{aligned}
& F(-\Lambda, \theta)=1-h+\sum_{j=1}^{\infty} c_{1 j} \cos (j \theta), \\
& F(\Lambda, \theta)=1+h+\sum_{j=1}^{\infty} c_{2 j} \cos (j \theta),
\end{aligned}
$$

\begin{tabular}{|c|c|}
\hline Wriphc & 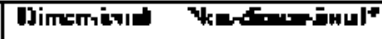 \\
\hline mial aoxditen & $2 \quad ;-\frac{d \quad 16,6=1 !}{k_{0}}$ \\
\hline Iudun & 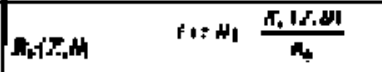 \\
\hline 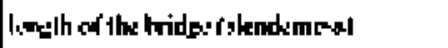 & $1.0 \mathrm{ER}$ \\
\hline 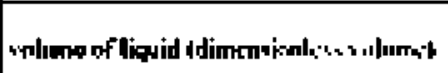 & $\mathrm{r}^{+} \quad: \frac{d_{+}}{\sin ^{*}}$ \\
\hline aial load (land numberl & $n-\frac{a k i}{\pi}$ \\
\hline
\end{tabular}

where, $h=\left(R_{20}-R_{10}\right) /\left(R_{20}+R_{10}\right)$, so that $R_{20} / R_{0}=1+h$, $R_{10} / R_{0}=1-h$, and $c_{i j}=C_{i j} / R_{0}$ measure a generic ripple term in the left $(i=1)$ or right $(i=2)$ side.

Table 1. Change from dimensional to non-dimensional variables in the molten zone. In parenthesis the name of the corresponding dimensionless variable.

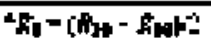


The formulation is completed with the conditions of azimuthal periodicity and volume preservation:

$$
\begin{aligned}
& F(z, \theta+2 \pi)=F(z, \theta), \\
& \frac{1}{2} \int_{-\Lambda}^{\Lambda} d z \int_{0}^{2 \pi} F^{2} d \theta=2 \pi \Lambda V .
\end{aligned}
$$

where $\Lambda$ is the slenderness or aspect ratio. Assuming nearly cylindrical interfaces for any slenderness, the problem is defined by the set of small parameters $\left\{v, h, B, c_{1 j}, c_{2 j}\right\}$, where: $v=V$ - 1. We take as the configuration of reference to be perturbed a cylindrical-volume liquid-bridge ( $v=V-1=0$ ), held between coaxial circular disks $\left(c_{i j}=0\right)$, equal in diameter $(h=0)$, and in absence of body forces $(B=0)$. The configuration of reference, the set $\left\{v=0, h=0, B=0, c_{1 j}=0, c_{2 j}=0\right\}$, corresponds to a perfect cylindrical bridge, and has the well-known trivial solution $F=1, P=1$. If now we consider a situation where the different parameters under consideration are not zero, but small, the expression for the liquid bridge interface can be expanded in the above quoted parameters, as follows:

$$
\begin{aligned}
& F(z, \theta)= \\
& 1+\sum_{i=i}^{2} \sum_{j=1}^{\infty} \varepsilon_{i j} f_{i j}(z, \theta)+\ldots, \quad P=1+\sum_{i=i}^{2} \sum_{j=1}^{\infty} \varepsilon_{i j} p_{i j}+\ldots
\end{aligned}
$$

where $\varepsilon_{i j}$ stand for the different small parameters: $\varepsilon_{01}=v, \varepsilon_{02}=$ $B, \varepsilon_{03}=h$ (and $\varepsilon_{0 j}=0$ for $j>3$ ) $\varepsilon_{1 j}=c_{1 j}, \varepsilon_{2 j}=c_{2 j}, \ldots$ The introduction of these asymptotic expansions into expression (5 - 10) yields, after neglecting terms of order $\mathcal{E}^{2}$ and higher, the following $\varepsilon$-order problems:

$$
\begin{aligned}
& \frac{\partial^{2} f_{i j}}{\partial z^{2}}+\frac{\partial^{2} f_{i j}}{\partial \theta^{2}}+f_{i j}+p_{i j}-\delta_{i j, i 2} z=0, \\
& f_{i j}(-\Lambda, \theta)=-\delta_{i j, 03}+\delta_{i j, 1 j} \cos (j \theta), \\
& f_{i j}(\Lambda, \theta)=\delta_{i j, 03}+\delta_{i j, 2 j} \cos (j \theta), \\
& f_{i j}(z, \theta+2 \pi)=f_{i j}(z, \theta), \\
& \int_{-\Lambda}^{\Lambda} d z \int_{j}^{2 \pi} f_{i j} d \theta=2 \pi \Lambda \delta_{i j, i 1},
\end{aligned}
$$

with $\delta_{i j, k l}=1$, if $i j=k l$, and $\delta_{i j, k l}=0$ if $i j \neq k l$.

The above problem formulation gives the following particular solutions:

$$
\begin{aligned}
& f_{01}=\frac{1}{2} \frac{\Lambda(\cos z-\cos \Lambda)}{\sin \Lambda-\Lambda \cos \Lambda}, \\
& f_{i 2}=z-\Lambda \frac{\sin z}{\sin \Lambda}, \\
& f_{i j}=\frac{\sin z}{\sin \Lambda}, \\
& f_{11}=\frac{\Lambda-z}{2 \Lambda} \cos (\theta), \\
& f_{1 j}=\frac{\sinh \left((\Lambda-z) \sqrt{j^{2}-1}\right)}{\sinh \left(2 \Lambda \sqrt{j^{2}-1}\right)} \cos (j \theta), \text { for } j>1, \\
& f_{21}=\frac{\Lambda+z}{2 \Lambda} \cos (\theta), \\
& f_{2 j}=\frac{\sinh \left((\Lambda+z) \sqrt{j^{2}-1}\right)}{\sinh \left(2 \Lambda \sqrt{j^{2}-1}\right)} \cos (j \theta), \text { for } j>1 .
\end{aligned}
$$

For the composite solution we only retain the first term in the last series and apply it to the near-square case, i.e. $j=4$. Therefore:

$$
\begin{aligned}
& F(z, \theta)=1+v f_{01}+B f_{02}+h f_{03}+c_{14} f_{14}+c_{24} f_{24} \\
& =1+v \frac{1}{2} \frac{\Lambda(\cos z-\cos \Lambda)}{\sin \Lambda-\Lambda \cos \Lambda}+B\left(z-\Lambda \frac{\sin z}{\sin \Lambda}\right)+h \frac{\sin z}{\sin \Lambda} \\
& +c_{1} \frac{\sinh (\sqrt{15}(\Lambda-z))}{\sinh (2 \sqrt{15} \Lambda)} \cos (4 \theta)+c_{2} \frac{\sinh (\sqrt{15}(\Lambda+z))}{\sinh (2 \sqrt{15} \Lambda)} \cos (4 \theta)
\end{aligned}
$$

The $i j=01,02$ and 03 first-order problems had been already solved [13]. Note that in the above expression $f_{01}$ is symmetric with respect to the middle plane parallel to the disks (that means that $f(z)=f(-z)$ ), whereas the others $\left(f_{02}\right.$ and $\left.f_{03}\right)$ are antisym- 
metric $(f(z)=-f(-z))$. In consequence, at least within the range of validity of this linear analysis, any variation of the liquid bridge volume with respect to the cylindrical volume causes a symmetric deformation of the liquid bridge interface (interface bulging or necking at the centre of the liquid column is obtained depending on the sign of $v$ ). On the other hand the interface deformation due to both the axial Bond number and the radii ratio of the supporting disks, causes the bulging of one half of the liquid column and the necking of the other, and since the deformations due to each one of the parameters involved, $h$ and $B$, can be in phase or in counter-phase, in the former case the necking effect will be accentuated (that means to be closer to stability loss), whereas in the second, both effects can compensate or even cancel each other. Therefore, liquid bridges between unequal disks are less stable when gravity points towards the larger disk than when it points towards the smaller one [14].

\section{3 Approximation of the shape of the grown crystal}

Here the evolution of the external shapes during the process of crystal growth is simulated, instead of by giving some heating and pulling laws and a furnace model, by giving explicit laws of motion of the two solid interfaces, and assuming a constant growth angle, $\phi_{\mathrm{m}}$, only dependent on the material (e.g. $11^{\circ}$ for silicon, $13^{\circ}$ for germanium). Although in normal operation one end melts and the other solidifies, the possibility of both ends being melting fronts (at the beginning of the process) or both being solidifying (at the end, when the furnace is stopped) is also considered.

Mathematically, the idea is to provide boundary conditions to integrate the liquid interface shape (11-16), or to build the linear approximation to the integrated shape (22) at every stage, although these boundary conditions are also differential equations since it is a moving boundary-value problem.

If only the first harmonic is retained, the non-dimensional shape of the liquid interface (22) has six parameters, namely the set $\left\{\Lambda, v, B, h, c_{1}, c_{2}\right\}$, where subscript 1 and 2 refer to the seed and feed sides of the melt. Starting with known values, e.g. $\{\Lambda$ $\left.=\Lambda_{\text {ini }}, v=v_{\text {ini }}, B=B_{\text {ini }}, h=0, c_{1}=c_{1, \text { ini }}, c_{2}=c_{2, \text { ini }}\right\}$, and for given speeds of the solid fronts, $d Z_{1} / d t$ and $d Z_{2} / d t$ (that might be dependent on time), the growing equations in physical variables are (see Fig. 2):

$$
\begin{aligned}
& \frac{d L}{d t}=\frac{d Z_{2}}{d t}-\frac{d Z_{1}}{d t} \\
& \frac{d V_{F}}{d t}=\left(-\pi R_{10}^{2} \frac{d Z_{1}}{d t}+\pi R_{20}^{2} \frac{d Z_{2}}{d t}\right) \frac{\rho_{s}}{\rho} \\
& \frac{d g}{d t}=0
\end{aligned}
$$

if growing at 1 -end $\left(d Z_{1} / d t>0\right)$

$$
\arctan \left(\frac{\partial R_{S 1}(Z, \theta)}{\partial Z}\right)_{Z=Z_{1}}=\arctan \left(\frac{\partial F(z, \theta)}{\partial z}\right)_{z=-\Lambda}-\phi_{m}
$$

if not growing at $1-$ end $\left(d Z_{1} / d t<0\right)$

$$
F(-\Lambda, \theta)=\frac{R_{S 1}\left(Z_{1}, \theta\right)}{R_{0}}
$$

if growing at 2 -end $\left(d Z_{2} / d t<0\right)$

$$
\arctan \left(\frac{\partial R_{S 2}(Z, \theta)}{\partial Z}\right)_{Z=Z_{2}}=\arctan \left(\frac{\partial F(z, \theta)}{\partial z}\right)_{z=\Lambda}+\phi_{m}
$$

if not growing at 2-end $\left(d Z_{2} / d t>0\right)$

$$
F(\Lambda, \theta)=\frac{R_{S 2}\left(Z_{2}, \theta\right)}{R_{0}}
$$

In the above expressions $\rho_{S}$ and $\rho$ are the solid and liquid densities, and the fact that $\partial R_{F} / \partial Z=\partial F / \partial z$ has been used. The two solid ends evolve independently, thus $R_{S 1}(Z, \theta)$ and $R_{S 2}(Z, \theta)$ enter into the problem, although from now on we assume that only the left side is growing and thus changing shape.

Approximating the trigonometric function in (26) by their

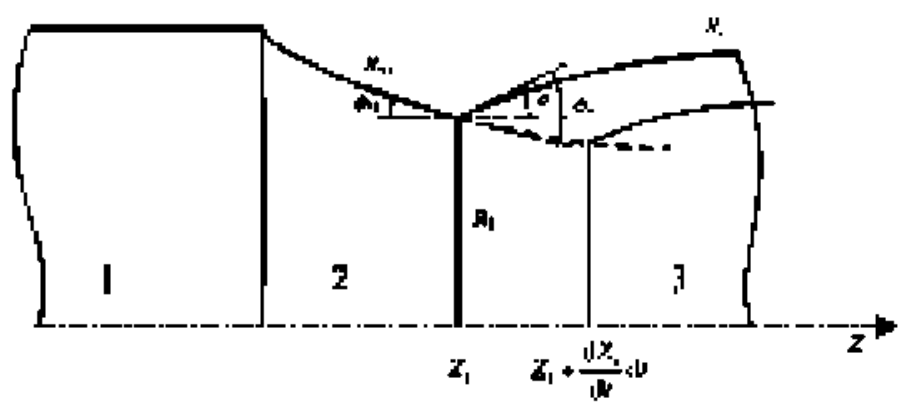

Fig. 2. Detail of growth angle geontery. A given jump between the slope of the growing crystal, $\phi_{S 1}=$ arctan( $\left.\partial R_{S I} / \partial Z\right)$, and the slope of the mett, $\phi=$ arctan $\partial F / \partial_{2}$ ), is maintained (constant growth angle, $\phi_{m}$ ). The advancing step used in the numerical simulation is exaggerated. 1 , seed: 2 , grown crystal; 3 , melt. 
arguments, and substituting the linear expression for $F(z, \theta)$ giving in (22), equation (26) becomes:

$$
\begin{aligned}
& \frac{d R_{S 1}}{d t}=\frac{d Z_{1}}{d t}\left(\left.v \frac{d f_{01}}{d z}\right|_{z=-\Lambda}+\left.B \frac{d f_{02}}{d z}\right|_{z=-\Lambda}+\left.h \frac{d f_{03}}{d z}\right|_{z=-\Lambda}\right. \\
& \left.+\left.c_{1} \frac{\partial f_{i 44}}{\partial z}\right|_{i=-\Lambda}+\left.c_{2} \frac{\partial f_{5}}{\partial z}\right|_{i=-\Lambda}-\phi_{m}\right)
\end{aligned}
$$

although what matters is not the whole variation of any radial position during the growth, but just the variation of the mean radius and the first harmonic, $d R_{10} d d t$ and $d C_{14} / d t$, that from (3) and (30) become:

$$
\begin{aligned}
& \frac{d R_{10}}{d t}= \\
& \frac{1}{2 \pi} \int_{0}^{2 \pi} \frac{d R_{S 1}}{d t} d \theta \\
& =\frac{d Z_{1}}{d t}\left(\left.v \frac{d f_{01}}{d z}\right|_{z=-\Lambda}+\left.B \frac{d f_{02}}{d z}\right|_{z=-\Lambda}+\left.h \frac{d f_{03}}{d z}\right|_{z=-\Lambda}-\phi_{m}\right) \\
& \frac{d C_{14}}{d t}=\frac{1}{\pi} \int_{i}^{2 \pi} \frac{d R_{S 1}}{d t} \cos (4 \theta) d \theta \\
& \left.=\left.\frac{d Z_{1}}{d t}\left(\frac{f_{14}}{\cos (4 \theta)}\right)\right|_{z=-\Lambda} ^{d z}+\left.c_{2} \frac{d\left(\frac{f_{24}}{\cos (4 \theta)}\right)}{d z}\right|_{\bar{z}=-\Lambda}\right)
\end{aligned}
$$

To simulate the growing process, the iterative procedure is as follows: with the initial molten state obtained, take a convenient time increment $\Delta t$ and compute the new geometry with (23), (24), (31) and (32), all in dimensional real variables; thence change to non-dimensional variables using Table 1 and recompute the new molten shape, and iterate until the desired crystal length is grown or an instability is found, either because the liquid shape appears to neck too much near the solidification front forced by a grown crystal necking, or because the liquid shape appears to neck too much near the melting front forced by liquid depletion due to a fat growing crystal. For simplicity, in this work we assume that a necking beyond $50 \%$ of the mean radius indicates subsequent breakage of the molten bridge, but the exact stability limit could be found by applying the well- known liquid bridge stability theory [14]. The practical difference between the two approaches is most of the times negligible.

To make it more explicit, an example is numerically simulated with the above algorithmic formulation and presented below. This procedure may be compared with the fully analytical simulation made in [15] for the related problem of an axisymmetric growth.

\section{Application to the case of growing from a nearly-square bar}

Consider an initially near-square bar (of uniform cross-section) of raw solid material, defined by its mean radius $R_{i 0}$ (e.g. $R_{i 0}=$ $5 \mathrm{~mm}$ ) and $C_{i 4} / R_{i 0}=0.14$ (the first coefficient of the Fourier cosine-expansion of a square). If the figure-of-merit for "squareness" is defined by the ratio of the area of the inscribed square to the area of the whole cross-section, a circle has a ratio of $2 / \pi$ $=0.63$, whereas with the fist harmonic it goes up to 0.95 . To better grasp how much square the first harmonic gives, Fig. 3a shows the whole cross-section, but in Fig. $3 b$ only two meridian cuts are shown: $R_{\theta=0}$ (i.e. maximum radii) and $R_{\theta=\pi^{\prime 4}}$ (i.e. minimum radii). In the figures to follow, only these two radial profiles are used to visualise the $3 \mathrm{D}$-shape in $2 \mathrm{D}$ plots.

Consider that a certain length $L$ of this bar has been molten and assume that this is really the initial state from which crystal growth is to be simulated. The initial liquid interface is found from Eq. (22) and plotted in Fig. $4 \mathrm{a}$ for the cases of $L /\left(2 R_{1}\right)=1$ and two values of axial gravity, corresponding to $B=0$ and $B=$ 1 ; in this case, this large Bond value is still acceptable for the linear analysis of short liquid bridge (in a recent review [7] of the hydrostatics of liquid bridges with several loads, it can be seen that the maximum Bond value for the present geometry is larger than 2). The $8 \%$ volume reduction upon melting for silicon growth has been accounted for and can be clearly appreciated in the figure. To see the effect of non-equal initial supports, Fig. 4b has been computed to show the initial molten shape between a near-square and a perfect circular support of different sizes $\left(R_{10} / R_{20}=0.8 / 1.2, L /\left(R_{10}+R_{20}\right)=1\right.$. Observe that, in any case, the non-axisymmetric effects only count near the non-circular ends, the liquid bridge tending to be axisymmetric in mid lengths.

Now the simulated crystal growth process may be started by considering some pulling-heating law equivalent to, say, a growing rate of $d Z_{1} / d t=10^{-5} \mathrm{~m} / \mathrm{s}$ and constant molten length $d Z_{2} / d t$ $=d \mathrm{Z}_{1} / d t$ (for constant length evolution), and an adequate time step for the discrete simulation (say $\Delta t=10 \mathrm{~s}$ ), irrelevant for the physics being simulated. Some results are presented in Fig. 5 , simulating the crystal growth from a nearly-square silicon rod with a molten zone of aspect ratio $\Lambda=0.5$ (Fig. 5a), $\Lambda=\mathrm{I}$ (Fig. 5b), and $\Lambda=2$ (Fig. 5c), without external loads ( $B=0$ ). It can be seen how the $11^{\circ}$ growth-angle for silicon (Fig. 2) tends to neck the solid grown. Although the molten length, material properties and applied loads are kept constant in the three series, 

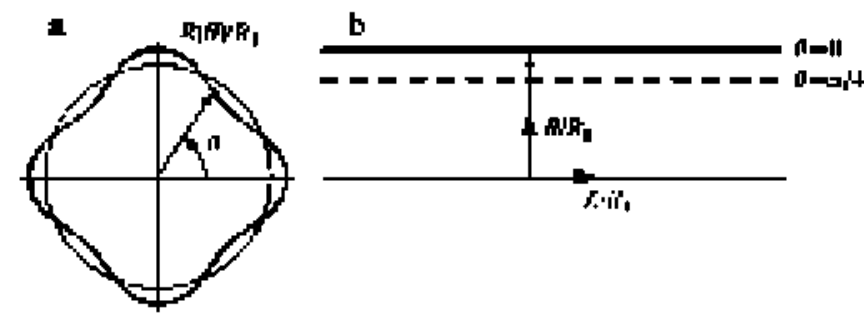

Fig. 3. Initial near-square solid bar in units of its mean radius $R_{I}$ : a) whole cross-section, b) only two meridian cuts are shown: $R(O) / R_{I}$ (i.e. maximum radii, solid line) and $R(\pi / 4) / R_{I}$ (i.e. minimum radii, dash line). In the figures to follow, only these two radial profiles are used to vistalise the $3 D$-shape in $2 D$ plots.
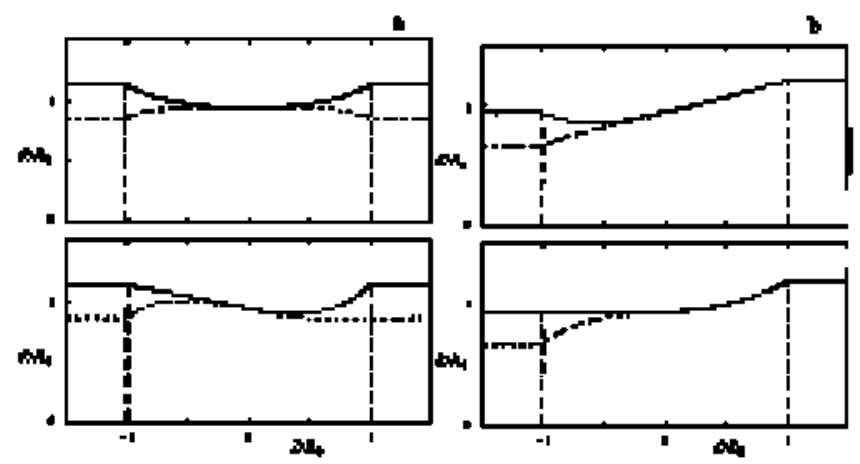

Fig. 4. Initial mollen shape of slenderness $A=L\left(2 R_{0}\right)=1$ of the bar in Fig. 3 (the two extreme meridians shown, for $\theta=0$ solid, and for $\theta=\pi / 4$ dashed) for the case of zero gravity (upper) and for an axial gravity corresponding to $B=$ $I$ (lower), in units of the mean radius $R_{0}$. the more slender one ( $\Lambda=2$ ) shows unstable growth (neck breaking will follow). In any case, both the grown solid and the molten zone tend to be axisymmetric, with the non-axisymmetry limited to the ends.

The maximum slenderness of the molten zone for stable crystal growth depends on the applied loads, and in Fig. 6 the stable limits in the case of a constant axial load (residual acceleration) is presented as a function of the slenderness, for the initially near-square bar. The upper limit of stability in Fig. 6 corresponds to the molten bridge necking near the melting front (with fat crystal growing), and the lower limit to the molten bridge necking near the solidification front (with narrowing crystal growth).

To better appreciate the effect of an axial residual gravity on the growth and its stability, Fig. 7 has been prepared by simulation of a floating zone process of constant molten length, $\Lambda=2$, in an initially near-square silicon bar, for the three loads corresponding to the three regions in Fig, 6: an axial load $B=0.040$ in the middle of the stable region, and two unstable loads, $B=$ 0.010 and $\mathrm{B}=0.075$, one at each side of the stability limits (check with Fig. 6).

As said before, although most of the results here presented refer to an initial prismatic silicon bar with a near-square crosssection, the procedure here developed allows for any geometry of the end supports: unequal seed and feed sides, even eccentric, with the only bounds of the linear analysis based on the cylindrical geometry, that is for radial departures much smaller than the mean radius.
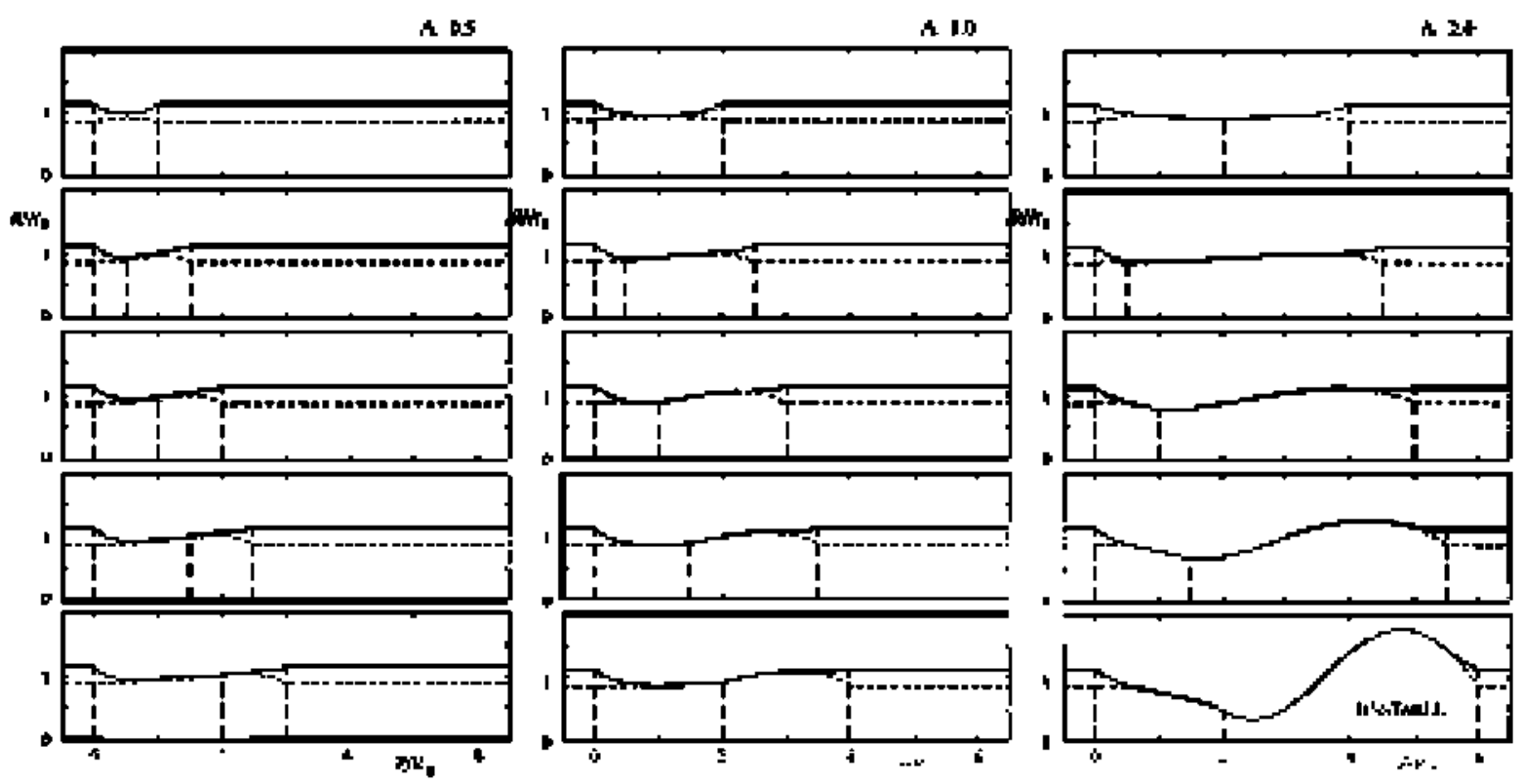

Fig. 5. Simulated crystal growth from an initially near-square silicon bar of mean radius $R_{0}$ and aspect ratio $A=0.5,1$ and 2 , without external loads $; B=0$, where it can be seen how the $11^{\circ}$ growth-angle (Fig. 2) tends to neck the solid grown. 


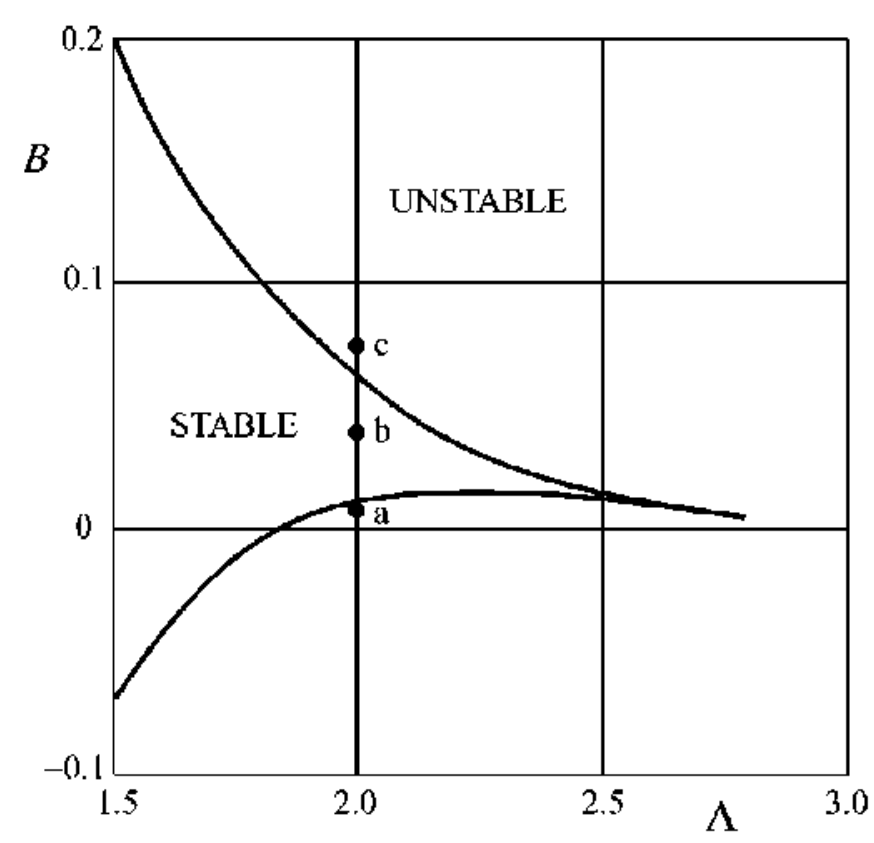

Fig. 6. Limiting axial loads (measured by the Bond number, B) for stable crystal growth from an initial liquid bridge obtained by zone-melting in a nearsquare silicon bar of mean radius $R_{0}$, in the case of maintaining a constant molten length $L$. Growth shapes at $a, b$ and $c$ depicted in Fig. 7.

\section{Conclusions}

The objective of this paper was to simulate the growing of crystals of non-circular cross-section by the floating zone technique and analyse the sequence of shapes and their stability. This has been done by using the hydrostatic liquid-bridge model (without any internal motions), shown in the literature to be representative of the mechanical behaviour in the real process.

The simulation has been achieved by a mathematical model valid for shapes not too far from that of a cylindrical molten zone. As an example, it has been applied to an initially nearsquare silicon bar, with an initial departure from the mean cylinder of $14 \%$ to best approach a square cross-section, expecting an uncertainty in the results of the order of a few percent.

As a general result, it has been found that there is a strong tendency for the liquid interface to get circular, and subsequently for the grown solid to get a circular cross-section, even if the initial rod is not of circular cross-section.

Detailed results have been found for the maximum molten length allowing a stable growth (without necking and bridge rupture) as a function of the residual axial gravity (measured by the Bond number).

The algorithm developed could be directly applied to simulate the growth with different geometries and different growth laws, within the linearised mathematical model.

As a follow-on, a more closed-form analysis might be pur-

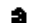

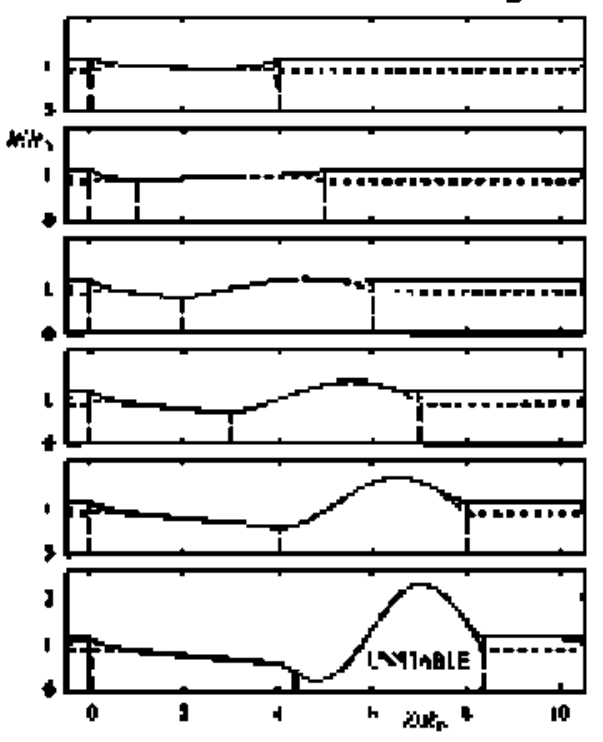

b

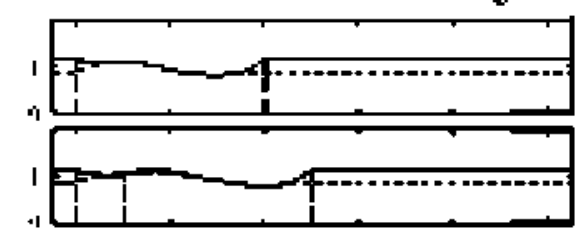

min.

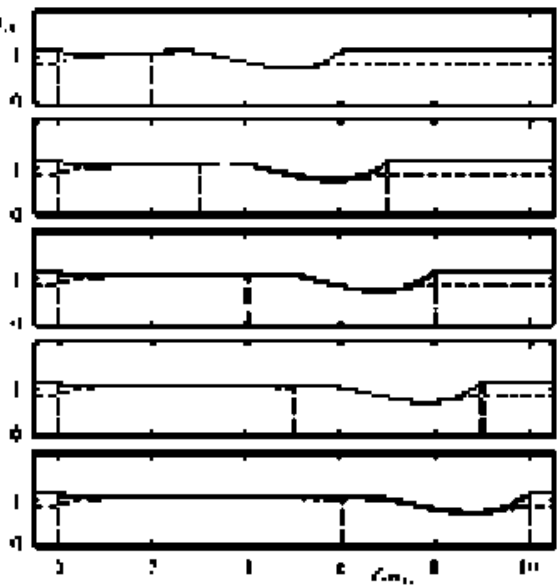

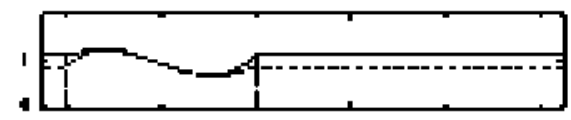

Ming
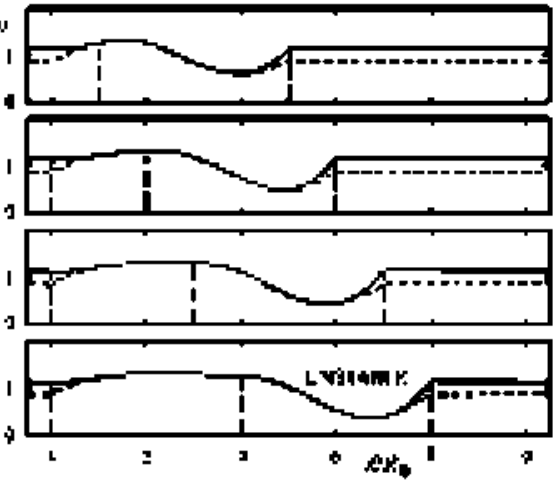

Fig. 7. Simulated crystal growh from an initially near-square silicon bar of mean radius $R_{0}$ assuming a fumace control law such as to keep the molten length $L$ constan all the time with $A=2$, corresponding to the three cases marked in Fig. 6 : axial loads $B=+0.010(a), B=+0.0$ to (b) and $B=+0.075$ (c), the middle one being the only stable grown. 
sued, for instance to develop an analytical expression for the limits of stable growth as a function of the geometry and the loads, or a more applied numerical simulation of the non-linear aspects (geometries far from the cylinder, large shape deformations, and so on).

\section{References}

1 Lan $C . W$, Kou, S.: "Shortened floating zone crystal growth under normal gravity". J. Crystal Growth 119, 281, 291 (1992).

2 Cröl, A., Kaiser, T., Schweizer, M., Danilewsky, A. N., Lauer, S., Tegetmeier, $A$, and $B e n z, K, W$ : "Floating-zone and floating-solution-zone growth of GaSb under mictogravity". J. Crystal Growth 191, 365, 376 (1998).

3 Martinez, I., Croll, A.: "Liguid bridges and floating zones". ESA SP-333, 135,142 (1992)

4 Martinez, I., Eyer, A.: "Liquid bridge analysis of silicon crystal growth expetiments under microgravity". J. Crystal Growth 75, 535, 544 (1986).

5 Boucher, E. A.,Evans, M. J.B.: "A liquid-bridge motel for the float-zone processing of materials". J. Chem. Soc. Faraday Trans. I. 81, 2787, 2796 (1985).

6 Martinez, I., Perales, J. M.: "Mechanical behaviour of liquid bridges in mictogravity in Physics of Fluids in Microgravity". in "Physics of Fluids in Microgravity", R. Monti (Ed.), Gordon \& Breach, (to appear in 2002).

7 Meseguet, J., Perales, J. M., Martínez, I., Bezdenejnykh, N. A., Sanz A.: "Hydrostatic Instabilities in Floating Zone Crystal Growth Process". Current Topics in Crystal Growth Research 5, 27, 42 (1999).

8 Rossolenko, S. N., Zhdanor, A. V.: "Equilibrium shapes of liquid menisci subjected to gravity force and surface tension". J. Crystal Growth 104, 8, 13 (1990).

9 Petkov, I. S., Red'kin, B. S.: "Calculation of the stationary ctoss-section form of nonaxisymmetrical ctystals growing from the melt". J. Crystal Growth $131,598,606$ (1993).

10 Tatarchenko, V. A., Uspenski, V. S., Tatarchenko, E. V., Nobot, I. Ph., Duffat, T., Roux, B.: "Theoretical model of crystal growth shaping process". J. Crystal Growth 180, 615, 626 (1997).

11 Hanidi, M., Rodot, H.: "Experimental determination of the growth angle: application to silicon". Revue de Physique Appliquée 18, 75, 80 (1983).

12 Martinez, I, Sanz A., Perales, J. M., Meseguer, J.: "Freezing of a Long Liquid Column on the Texus-18 Sounding Rocket Flight". ESA Journal 12, 483. 489 (1988).

13 Bezdenejnykh, N. A., Meseguer, J.: "On the use of liquid bridges as accelerometers". Microgravity Sci. Technol. 9. 62. 69 (1996).

14 Perales, J. M. Meseguer, J., Martinez I.: "Minimum Volume of Axisymmetric Liquid Bridges between Unequal Disks in an Axial Microgravity Field". J. Crystal Growth $110.855,861$ (1991).

15 Martinez I, Meseguer, J., Perales, J. M.: "Analytical Modelling of Floating Zone Crystal Growth". Adv. Space Res. (to appear in 2002).

\section{Acknowledgements}

This work has been supported by the Spanish Ministerio de Ciencia y Tecnología (MCYT). 